This is a postprint version of the following published document:

Cabanelas, J. C., Serrano, B. \& Baselga, J. (2005). Development of Cocontinuous Morphologies in Initially Heterogeneous Thermosets Blended with Poly(methyl methacrylate). Macromolecules, 38 (3), pp. 961-970.

"This document is the unedited Author's version of a Submitted Work that was subsequently accepted for publication in Macromolecules, copyright (C) 2005 American Chemical Society after peer review. To access the final edited and published work see http://pubs.acs.org/doi/abs/10.1021/ma0487352 or see DOI: $\underline{10.1021 / \mathrm{ma} 0487352 "}$ 


\title{
Development of Cocontinuous Morphologies in Initially Heterogeneous Thermosets Blended with Poly(methyl methacrylate)
}

\author{
J. C. Cabanelas, ${ }^{*}$ B. Serrano, ${ }^{\dagger}$ and J. Baselga ${ }^{\ddagger}$
}

Department of Materials Science and Engineering and Chemical Engineering, Universidad Carlos III de Madrid, 28911 Leganés, Spain

\begin{abstract}
Morphology and phase separation process in blends of a network-forming reactive polymer, poly(aminopropylmethylsiloxane) (PAMS), in a poly(methyl methacrylate) (PMMA)-modified epoxy system were studied using optical, epifluorescence and scanning electron microscopy, Fourier transform infrared spectroscopy (FTIR), and dynamic mechanical thermal analysis (DMTA). The thermoset system was bisphenol A diglycidyl ether (DGEBA) with different PMMA percentages, $2-10 \% \mathrm{w} / \mathrm{w}$. Phase separation and reaction advancement were monitored in situ. At the concentration studied, PMMA does not influence the kinetics of the curing process, but it strongly affects the reactive compatibilization between DGEBA and PAMS. The morphology obtained consists of a continuous thermoplastic-rich phase surrounding thermosetting connected polyhedral particles of 5-15 $\mu \mathrm{m}$. This cocontinuous morphology is observed independently of the percentage of PMMA. Results show that the morphology is strongly influenced by the diffusion and viscosity conditions during reactive compatibilization and phase separation. An increase in PMMA content leads to a decrease in the thermosetting polyhedral particle size. In contrast, an increase in curing temperature leads to bigger sizes. The addition of thermoplastic polymers to initially nonhomogeneous reactive blends is a potential route for generating cocontinuous morphologies irrespective of the thermoplastic content.
\end{abstract}

\section{Introduction}

Epoxy resins are widely studied materials because of their well-known cross-linking chemistry and good set of properties. Because of their three-dimensional molecular structure, epoxy resins give rise to high stiffness, high strength, and good heat and solvent resistance materials. ${ }^{1}$ These excellent properties make them frequently used in a large range of applications, from aerospace structures to dental fillers. However, the high level of cross-linking in epoxy networks leads to inherent brittle materials, and research points to the development of new engineering materials that could improve their properties. The incorporation of a thermoplastic (TP), initially miscible, which phase separates during the epoxy-hardener curing reaction, leads to toughness improved epoxy networks. A new material is generated with distinct properties with respect to those of the individual polymers. Thermoset/thermoplastic blends are currently being used as a means to toughen the resin network, improve mechanical resistance, reduce thermal stresses and modify their microstructure in a wide range of different morphologies, from evenly dispersed submicron-sized second phases to micropores. ${ }^{2-5}$

The morphology of the generated materials depends on the selection of the initial formulation-the thermoplastic polymer, the epoxy precursor and the hardenerand also on the selected curing cycle parameters. Particulate (TP on a continuous thermoset matrix), cocontinuous, or phase-inverted (thermoset particles on a TP matrix) structures can be achieved. The molar

\footnotetext{
* Corresponding author. Telephone: 34-91-6249469. Fax: 34-

91-6249430. E-mail: caba@ing.uc3m.es.

† Telephone: 34-91-6249469. Fax: 34-91-6249430. E-mail: berna@ing.uc3m.es.

† Telephone: $34-91-6249467$. Fax: 34-91-6249430. E-mail: jbaselga@ing.uc3m.es.
}

mass, TP weight fraction, reaction rate, and viscosity during phase separation bear a great influence on the final structure. The presence of the thermoplastic in a cocontinuous phase is a potential route for synthesizing, for example, porous thermosets ${ }^{6}$ or even conducting composites. ${ }^{7}$ What is more, if the added thermoplastic does not react with the network precursors, it is generally accepted that the fracture toughness improvement is more significant when cocontinuous or inverted structures are generated. ${ }^{2,4,8,9}$ These structures are usually obtained either by increasing the thermoplastic content beyond the critical value or by shifting the critical point to lower values increasing the thermoplastic molar mass, but they are not without their shortcomings, for example, poor solvent resistance and low creep properties. These drawbacks are usually solved by adding a reactive functionality to the thermoplastic polymer, ${ }^{2,10-12}$ although this solution forces synthetic routes that are not always easy or available for all thermoplastic-thermoset systems.

The development of the desired morphologies can also be achieved by modifying the factors that control phase separation behavior, such as the pseudobinary interaction parameter, viscosity, and conversion, all of them dependent on TP characteristics and on the molecular weight of the epoxy precursors at the cloud point. In previous works, we presented a novel oligomeric polyfunctional epoxy hardener, poly(3-aminopropylmethylsiloxane) (PAMS). ${ }^{13-15}$ This hardener has certain advantageous properties, such as, for example, low viscosity and good thermal resistance, both derived from the presence of the siloxane skeleton. On the other hand, the pendant aminopropyl aliphatic functional groups allow reaction with common epoxy resins, overlooking the traditional poor solubility of polysiloxanes in those systems, which usually leads to undesirable large domain sizes. ${ }^{3,16}$ In fact, PAMS is initially immiscible 
Table 1. Characteristics of the System Components

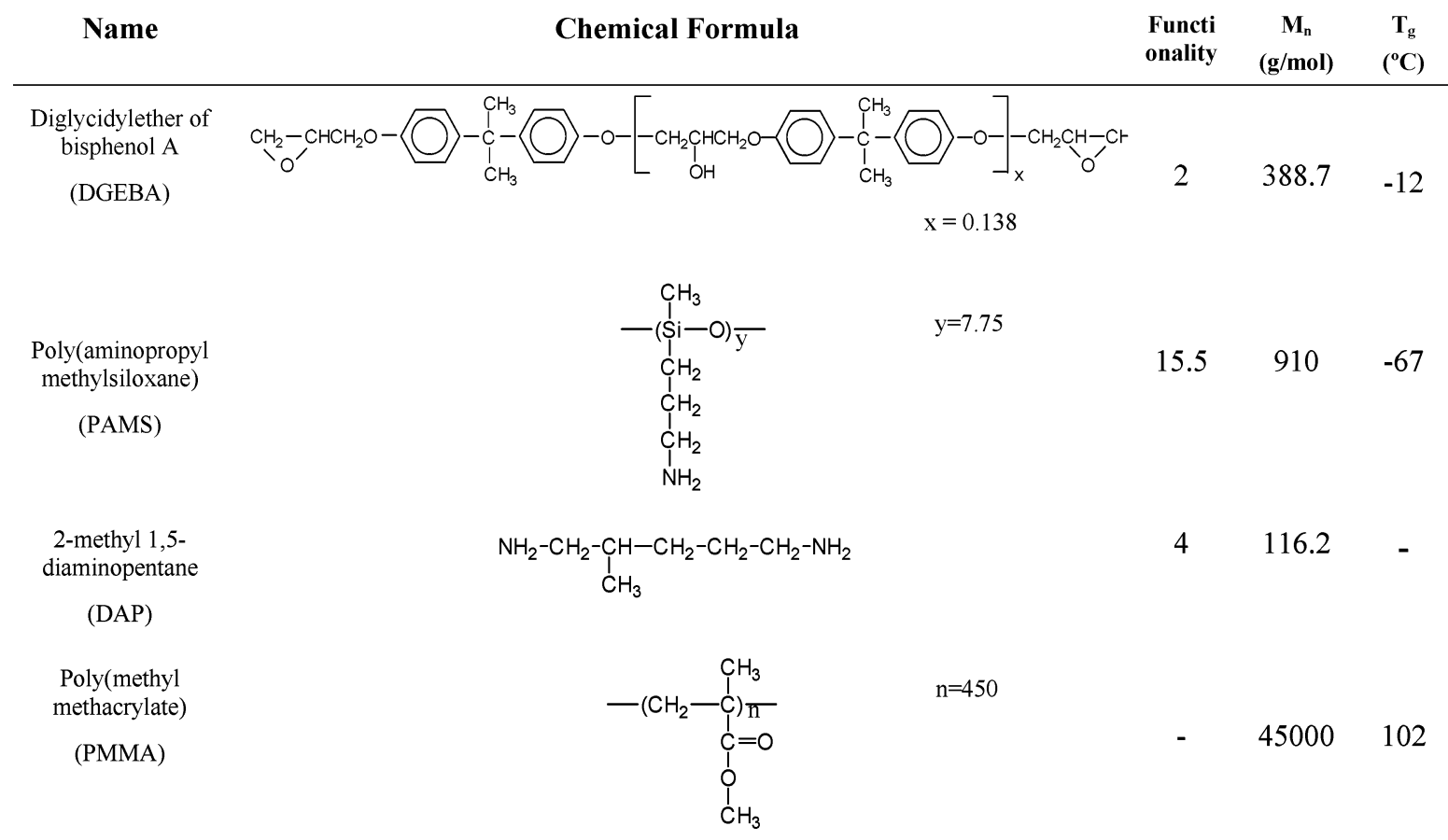

with DGEBA, but the system achieves fast solubilization by reactive compatibilization between PAMS and epoxy groups. ${ }^{14}$ The presence of a polyfunctional hardener introduces at least one new parameter for morphology control: conversion at the gel point. In fact, a polyfunctional hardener lowers gel conversion and prompts a faster increase of viscosity with curing, with unpredictable results when the TP phase separates.

In this study, poly(methyl methacrylate) (PMMA) was selected as the thermoplastic modifier to study the new morphologies generated during curing with PAMS as hardener. Although PMMA is not widely used as modifier because of its relatively low $T_{\mathrm{g}}$ in comparison with other engineering thermoplastics (e.g., PPE, PSF or PEI), this polymer has the unusual feature of being soluble in DGEBA in the full range of compositions. ${ }^{17-20}$ Several authors have reported solubility on the entire composition range as due to hydrogen bonding between the carbonyl group of PMMA and the hydroxyl groups of DGEBA. ${ }^{21}$ Like most TP modifiers, PMMA phase separates during curing when the incremented molar mass of the epoxy precursors involves a decrease in the conformational entropy of mixing. The generated morphologies range from particulate to phase-inverted structures depends on the hardener used.

The DGEBA/PAMS/PMMA system presents another interesting feature; common epoxy/diamine/TP reactive blends are usually treated as pseudobinary systems because the epoxy and the diamine components are miscible during the whole curing process. Because of the use of an immiscible hardener, the pseudobinary hypothesis can no longer be maintained. To the authors' knowledge, this type of system has not been reported on previously.

The aim of this study is to examine the behavior of PMMA/DGEBA blends when PAMS is used as hardener, at different curing conditions and different TP contents, between 0 and 10 wt \% on the DGEBA precursor. First, the influence of the TP on the curing process and on the reactive compatibilization of the thermoset precursors will be studied. Second, the phase separation process and the morphology of the cured blends will be analyzed and compared with those obtained using a conventional aliphatic diamine. As will be shown, a polyhedral morphology, which resembles the biliquid foams (aphrons) described by Sebba, ${ }^{22}$ as well as an explanation with respect to the formation of those structures, will be presented. Results will be compared with the model system DGEBA/2-methyldiaminopentane (DAP).

\section{Experimental Section}

Materials and Sample Preparation. Table 1 shows the chemical formulas and main characteristics of the monomers and polymers used in this study. The diglycidyl ether of bisphenol A resin (DGEBA) was supplied by GAIRESA (Spain), with a mass per mole of epoxy groups equal to $188.7 \mathrm{~g} \cdot \mathrm{mol}^{-1}$, determined by acid titration. The ratio of secondary hydroxyl groups to epoxy groups was 0.069 . Two different curing agents were used: 2-methyldiaminopentane (DAP), supplied by Aldrich and employed without further purification; and poly(3aminopropylmethylsiloxane) (PAMS), also provided by GAIRESA. PAMS was characterized by vapor pressure osmometry (VPO, Knauer K7000) and size exclusion chromatography (SEC, Shimadzu LC9A). $M_{\mathrm{n}}$ was $910 \pm 40 \mathrm{~g} \cdot \mathrm{mol}^{-1}$; the amine hydrogen equivalent was $58.5 \mathrm{~g} \cdot \mathrm{mol}^{-1}$. Polymer was stored in a desiccator to prevent amine carbonation prior to use.

The poly(methyl methacrylate) (PMMA) used in this work was labeled with a fluorescent reporter. Fluorescent labeling of PMMA was accomplished by radical copolymerization of methyl methacrylate with $1 \% \mathrm{w} / \mathrm{w}$ of 9 -vinylanthracene, in accordance with a method described previously. ${ }^{23}$ Characterization of PMMA was performed by SEC in THF using a set of PMMA standards (Polysciences), giving $M_{\mathrm{n}}=45000 \mathrm{~g} \cdot \mathrm{mol}^{-1}$ and a polydispersity ratio of 2.1 .

Solutions of PMMA (2-10\% w/w) in DGEBA were prepared by stirring both components at $110{ }^{\circ} \mathrm{C}$ up to complete dissolution; these solutions were clear as expected..$^{17,19,24}$ Curing was performed by mixing previously degasified appropriate amounts of the reactives, stirring at room temperature for $2 \mathrm{~min}$, and curing at desired temperature on the appropriate preheated device. The equivalent ratio epoxy/amine was maintained 
Table 2. Dependency on Temperature (K) of the Refractive Indices and Density of the Different Species Present in the Blends

\begin{tabular}{|c|c|c|c|c|}
\hline sample & $n\left(60^{\circ} \mathrm{C}\right)$ & $-\mathrm{d} n / \mathrm{d} T\left(\mathrm{~K}^{-1}\right)$ & $\begin{array}{l}\rho\left(25^{\circ} \mathrm{C}\right) \\
\left(\mathrm{g} \mathrm{cm}^{-3}\right)\end{array}$ & $\ln \rho$ \\
\hline PAMS & 1.461 & $2.67 \times 10^{-4}$ & 1.055 & $(0.284-7.595) \times 10^{-4} T$ \\
\hline DGEBA & 1.561 & $3.00 \times 10^{-4}$ & 1.170 & $(0.372-7.419) \times 10^{-4} T$ \\
\hline PMMA $^{a}$ & 1.487 & $1.2 \times 10^{-4}\left(T<T_{\mathrm{g}}\right) ; 3.1 \times 10^{-4}\left(T>T_{\mathrm{g}}\right)$ & 1.188 & $a$ \\
\hline
\end{tabular}

${ }^{a} \rho=(1.195-2.249) \times 10^{-4} \mathrm{~T}\left({ }^{\circ} \mathrm{C}\right)-1.943 \times 10^{-6} T^{2}\left({ }^{\circ} \mathrm{C}\right)$, taken from ref 41.

constant and equal to one in all cases. The selected curing temperatures were 60,90 , and $120^{\circ} \mathrm{C}$ and the PMMA content on the initial polymer/epoxy mixture was $0,2,5$, and $10 \mathrm{wt} \%$.

Measurements. Fourier transform infrared spectroscopy in the near range (FTIR, Perkin-Elmer GX2000, $4 \mathrm{~cm}^{-1}$ resolution) was employed to measure the extent of the epoxyamine reaction. After mixing, the sample was placed on a preheated sample cell composed of two glass slides and a Teflon spacer $(0.5 \mathrm{~mm})$. Epoxy conversion, $\alpha$, was determined following the extinction of the epoxy band $\left(4532 \mathrm{~cm}^{-1}\right)$ referred to a reference $\mathrm{C}_{\phi-\mathrm{H}}$ band $\left(4623 \mathrm{~cm}^{-1}\right)$, according to the following expression, where $S$ is the integrated absorbance of the band

$$
\alpha(t)=1-\frac{S_{4532}(t) / S_{4623}(t)}{S_{4532}(t=0) / S_{4623}(t=0)}
$$

A built-in optical device ${ }^{25}$ was used for measuring the cloud point. It consisted of an optical fiber coupling between a Fluorimeter (Perkin-Elmer LS50B) and a calorimeter (PerkinElmer DSC7). Transparency of the sample to $450 \mathrm{~nm}$ radiation was determined by the reflected light intensity from the aluminum pan of the DSC sample holder. This device is able to detect domains on the order of $0.2 \mu \mathrm{m}$.

Thermal transitions on cured samples were determined by measuring the loss factor $(\tan \delta)$ as a function of temperature using a dynamic mechanical thermal analysis instrument (DMTA, Rheometric Scientific Mk III, operating in a single cantilever bending mode at $3 \mathrm{~Hz}$ ). Temperature was increased at a rate of $2{ }^{\circ} \mathrm{C} / \mathrm{min}$. Sample dimensions were approximately $2 \times 6 \mathrm{~mm}$, with a gap setting of $5 \mathrm{~mm}$.

Blends morphology was studied by optical transmission microscopy (TOM), epifluorescence microscopy (EFM) and scanning electron microscopy (SEM). TOM observations (Olympus BHM) were carried out on samples prepared by putting a drop of resin between two glass slides and curing the sample in an oven at the desired temperature. Images were taken after quenching at room temperature. For the "in situ" study of morphology evolution during curing, the microscope was equipped with a hot stage (Leica VMTG) and a CCD camera. Epifluorescence and TOM simultaneous images were obtained under a different microscope (Nikkon Labophot) with a Nikkon camera, using a 100-W Hg arc lamp as source. The excitation beam was filtered with a band-pass filter to transmit in the range $330-380 \mathrm{~nm}$. The sample emission was separated from the excitation beam by a dichroic mirror (excitation wavelength $<400 \mathrm{~nm}$ ) and a barrier filter (emission wavelength $<420$ $\mathrm{nm})$. SEM observations were performed over $\mathrm{Au} / \mathrm{Pd}$ coated samples in a XL30 SEM microscope (Phillips).

Refractive indices were measured in a thermostated Abbe refractometer. DGEBA and PAMS densities were measured, at least at four temperatures, using a PAAR DMA602 Densimeter ( $5 \mathrm{~K}$ period) coupled to a thermostatic bath (temperature stability $\pm 0.02{ }^{\circ} \mathrm{C}$ ). Good linear fits were obtained on the logarithmic representation of $\rho$ vs $T$ over the whole range of temperatures used in this study. Both refractive index and density data are presented in Table 2 .

\section{Results and Discussion}

Influence of PMMA Content on the Curing Process. The progress of the curing reaction was followed by near range IR spectroscopy. An example of the collected spectra is presented in Figure 1. It was observed that both the epoxy (approximately $4532 \mathrm{~cm}^{-1}$ )

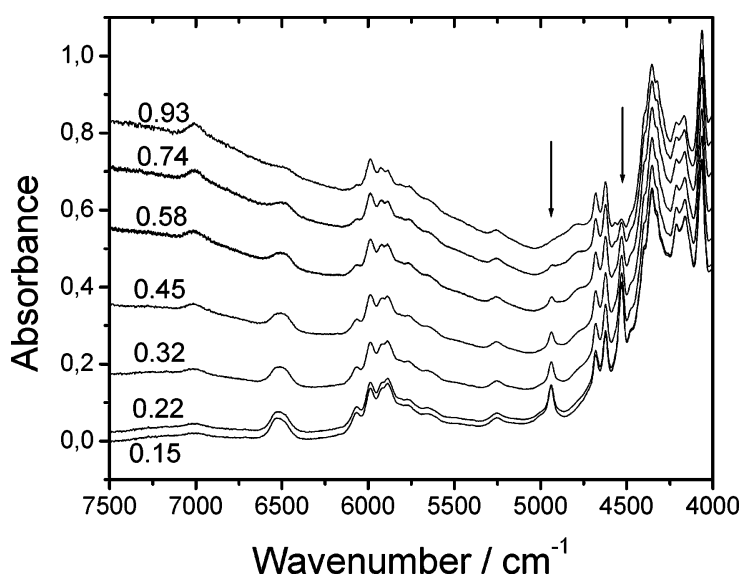

Figure 1. Infrared spectra obtained at different curing times for the system DGEBA/5\% PMMA/PAMS cured at $90^{\circ} \mathrm{C}$. The corresponding epoxy conversion is presented on the left side of each spectrum. Arrows mark the primary amine and epoxy bands.

and amine (approximately $4940 \mathrm{~cm}^{-1}$ ) bands clearly decreased with curing time and conversion could be calculated accordingly. Baseline variations were also observed and will be discussed further.

Figure 2 shows, as an example, the evolution of epoxy conversion with reaction time for DGEBA/X\% PMMA/ PAMS samples $(X=0,2,5,10)$, at curing temperatures of 60 and $90^{\circ} \mathrm{C}$. The system DGEBA/5\% PMMA cured with DAP is also included for comparison purposes.

It is noticeable that no variations on curing rate were observed when PMMA was added to the reaction system. There has been extensive debate in the literature about the effect of thermoplastic (TP) additions on the kinetics of epoxy systems. It has been reported that for relatively low TP content (around 10\% and above), the dilution effect on reactant concentration and increased viscosity induces a decrease in the curing rate. ${ }^{17,19,21,26}$ An apparent acceleration has also been observed in the reaction rate when the thermoplasticrich phase segregates. ${ }^{27}$ This accelerating effect has been attributed to the increased concentration of reactants in the epoxy-rich phase when TP segregation occurs. According to the conversion-time curves shown in Figure 2, neither dilution nor acceleration effects were present in our system. The TP concentration did not seem to influence polymerization kinetics. This could be attributed, in principle, to the low TP concentration of the samples, but this finding will be explained in greater depth below. As regards the comparison of the different samples, the very small variations in conversion-time were attributed to the inherent uncertainty of the FTIR technique. Therefore, a single averaged conversion-time curve, independent of PMMA content, can be calculated; this curve is also shown in Figure 2 .

The limiting conversion achieved for the PAMS cured systems, as calculated from the averaged curve, as well as for the DAP cured system, are presented in Table 3. It can be observed that when PAMS was used, lower 

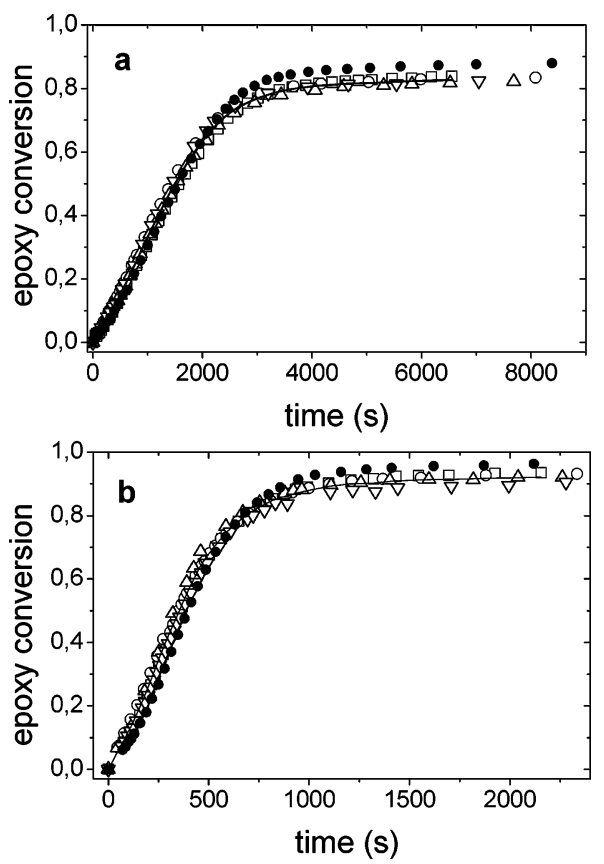

Figure 2. Conversion-time curves obtained in the system DGEBA/PAMS for neat $(O)$ and with $2 \%(\square), 5 \%(\triangle)$, and $10 \%$ $(\nabla)$ of PMMA on the epoxy precursor, at $60(\mathrm{a})$ and $90{ }^{\circ} \mathrm{C}(\mathrm{b})$. The continuous line (-) indicates the master curve performed from these four curves. Data from the DGEBA/DAP/5\% PMMA system are also included $(\bullet)$

Table 3. Limiting Conversion and Gel Time (According to Stockmayer Statistics) for DGEBA/PMMA/Hardener Blends $^{a}$

\begin{tabular}{ccrccc}
\hline $\begin{array}{c}T \\
\left({ }^{\circ} \mathrm{C}\right)\end{array}$ & hardener & $\begin{array}{c}\left(\omega_{\mathrm{TP}}\right)_{\mathrm{PMMA}} \\
(\%)\end{array}$ & $\begin{array}{c}\left(\phi_{\mathrm{TP}}\right)_{\mathrm{tb}} \\
(\%)\end{array}$ & \multicolumn{1}{c}{$\alpha_{\text {lim }}$} & $\begin{array}{c}t_{\text {rel }} \\
(\mathrm{min})\end{array}$ \\
\hline 60 & PAMS & 0 & 0 & $0.83 \pm 0.01$ & 13.5 \\
& & 2 & 1.45 & & \\
& & 5 & 3.65 & & \\
& DAP & 10 & 7.41 & & \\
90 & PAMS & 5 & 4.00 & 0.88 & 29.8 \\
& & 0 & 0 & $0.92 \pm 0.02$ & 3.33 \\
& & 2 & 1.43 & & \\
& & 5 & 3.62 & & \\
120 & DAP & 10 & 7.34 & & \\
& PAMS & 5 & 3.98 & 0.96 & 7.42 \\
& & 0 & 0 & $0.97 \pm 0.01$ & 1.20 \\
& & 2 & 1.43 & & \\
& & 5 & 3.60 & &
\end{tabular}

${ }^{\mathrm{a}}\left(\omega_{\mathrm{TP}}\right)_{\mathrm{DGEBA}}$ is the TP weight fraction in DGEBA resin, whereas $\left(\phi_{\mathrm{TP}}\right)_{\mathrm{tb}}$ corresponds to the TP volume fraction for the ternary blend.

limiting conversions were obtained in comparison with DAP, but the conversion-time curve profiles (Figure 2) were so similar that no relevant kinetic differences can be expected between both hardeners. This similarity confirms the suitability of selecting DAP for comparing results with PAMS. Nevertheless, an important difference between both arises from their different functionality. Table 3 also shows the theoretical gelation times and the TP volume fraction at the ternary mixtures. The first were obtained considering the Flory-Stockmayer approach. Gel conversion, which is 0.57 for DGEBA/ DAP, decreased to only 0.26 in DGEBA/PAMS systems because of its higher functionality. The TP volume fraction was calculated from the density data presented in Table 2.

Reactive Compatibilization and Transparency Window. The morphology in the first steps of the curing process can be explained in terms of the solubility of the three components of the mixture and can be interpreted from the behavior of the binary blends. Previous studies ${ }^{13,14}$ have shown that binary DGEBA/ PAMS blends are heterogeneous at the beginning and two phases are present. PAMS is the low-viscosity component, and it transforms into the continuous phase immediately after room temperature mixing. When heating to the curing temperature, phase inversion and coalescence of PAMS were observed, leading to a continuous epoxy-rich phase with dispersed PAMS-rich droplets. The driving force behind this behavior is curing temperature, as well as reaction kinetics, which changes the molecular weight distribution in the PAMS phase. Broad droplet distribution was observed, with a maximum size of about $20 \mu \mathrm{m}$. As curing progressed, the system reached reactive compatibilization by DGEBA diffusion through the PAMS domains. After curing (at $60{ }^{\circ} \mathrm{C}$ and above), the system appeared to be homogeneous: optical transparency, absence of clear interphases, and a single $T_{\mathrm{g}}$ were observed. ${ }^{14}$

As regards PMMA/PAMS blends, these presented quite different solubility parameters, $18.3 \mathrm{MPa}^{1 / 2}$ for PMMA and $19.5 \mathrm{MPa}^{1 / 2}$ for PAMS (obtained by the group contribution method), ${ }^{28}$ and these were immiscible for any composition. The reason seems also to be the high level of self-association of PAMS by strong hydrogen bonding. ${ }^{29}$

Therefore, at the curing temperatures used in this study, PMMA proved to be immiscible with PAMS but was completely miscible with DGEBA, and PAMS was initially immiscible with DGEBA. The mixture of the three components yielded a nontransparent initial solution. At the beginning of the curing reaction, DGEBA/ PMMA/PAMS blends consisted of at least two phases: DGEBA-PMMA rich matrix and PAMS-rich droplets, with broad droplet size distribution. In terms of the ternary blends containing DAP as hardener, these were always homogeneous from the outset of curing.

FT-nIR results enabled a brief analysis of the homogeneity of the blends. Following a method first applied by Koenig ${ }^{30}$ to mesophase transitions, the baseline changes in the FTIR spectra (Figure 1) were associated with changes in the blend transparency to the near-IR radiation. In fact, the baseline slopes observed in the FTIR spectra were due to variations in the scattering of the sample. The evolution of the baseline (taken as the absolute value at $6300 \mathrm{~cm}^{-1}$ ) with conversion is presented in Figure 3 for two curing temperatures. In the first stages of the curing of DGEBA/PMMA/PAMS samples, the baseline decreased to almost zero (maximum IR transparency), remaining at that value until a certain conversion level. In other words, the blend seemed to be homogeneous with respect to near-IR radiation. This finding may have two possible and excluding explanations: either an unlikely ternary solubility region was reached, at a given temperature and conversion; or the system turned into a multiphasic blend in which no differences were observed on the radiation scattering, i.e., equal refractive index in each phase. In any case, this behavior can be associated with the reactive compatibilization of the thermoset precursors. It should be noted that for the samples containing PMMA, the conversion at which this compatibilization occurred was higher than in samples not containing the thermoplastic. It therefore seems that, although it does not influence reaction kinetics, the presence of the TP delays reactive compatibilization. The DGEBA/PMMA/ 


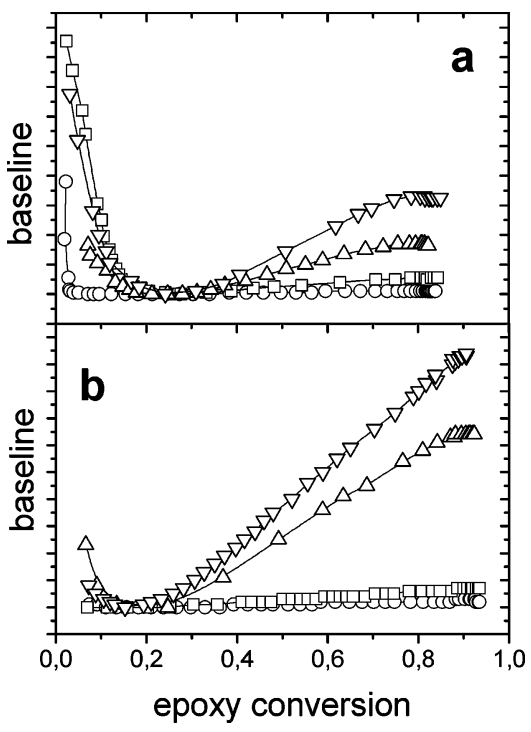

Figure 3. Baseline from FTIR spectroscopy as a function of epoxy conversion of DGEBA/PAMS for neat $(O)$ and with $2 \%$ $(\square), 5 \%(\triangle)$ and $10 \%(\nabla)$ of PMMA, at $60(\mathrm{a})$ and $90{ }^{\circ} \mathrm{C}(\mathrm{b})$.

DAP samples did not show this behavior because they remained homogeneous from the beginning of the reaction. Last, at a given epoxy conversion, baseline increased again in all the blends analyzed. This behavior could be associated, in principle, with the thermoplastic phase separation if the mixed blend were homogeneous.

To clarify the nIR results, the evolution of the reaction was followed "in situ" by TOM, as explained in the Experimental Section of this paper. Figure 4 shows, as an example, morphological evolution for the system DGEBA/5\% PMMA/PAMS with curing time. It was noticeable that optical microscopy was not able to detect any phase separated structures in the DGEBA/PMMA/ DAP samples.

The initial mixture of DGEBA/PMMA with PAMS, once heated at the curing temperature, was heterogeneous with a diffuse fine structure, as shown in Figure 4a. As the reaction progressed, this fine structure broadened (Figure 4b); in concordance with the abovementioned interpretation; broadening was the consequence of the interdiffusion of DGEBA and PAMS at the interfacial level. Suddenly, this fine structure disappeared and the system became optically homogeneous: this step corresponds to the entrance in the transparency window (Figure 4c) already observed by nIR. Shortly after a polygonal, "bubble-type", structure began to form and the sample once again became opaque (exit from the transparency window, Figure 4, parts $d$ and e, and no further changes appeared with time. The final structure (Figure 4f) was fixed even if a postcuring at $130{ }^{\circ} \mathrm{C}$ was performed. In Figure $4 \mathrm{~g}$ presents a SEM image of the surface, showing the final morphology; it reveals in great detail that their exist packed pseudo regular polygonal domains and also that the thickness of the thin interface or skin between them must be very small, less than $100 \mathrm{~nm}$.

Fluorescence labeling of the PMMA reveals the composition of the polygonal domains and the surrounding skin. Figure 5 shows a well-defined image of the morphology obtained for DGEBA/PMMA/PAMS samples. A clear two-phase structure is once again observed, in which polygonal domains, of a few micrometers in size, are regularly dispersed. To elucidate the phase composition, an epifluorescence image of exactly the same region

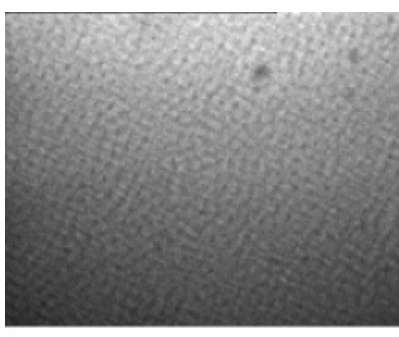

a

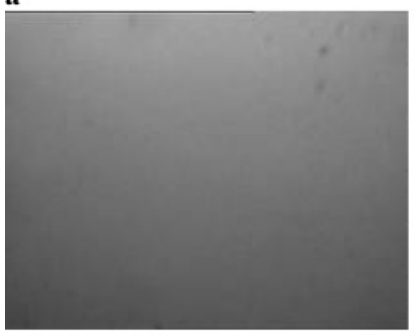

c

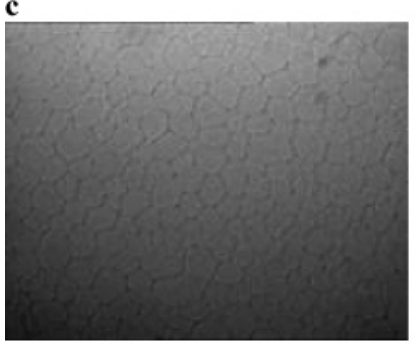

e

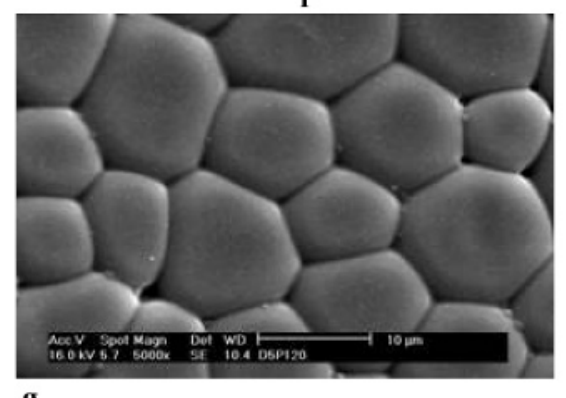

g

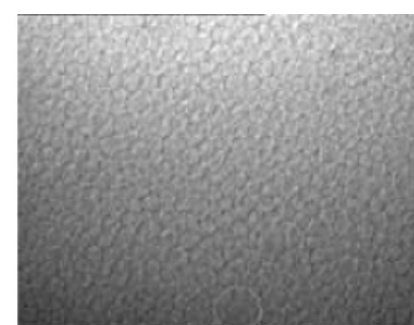

b

d

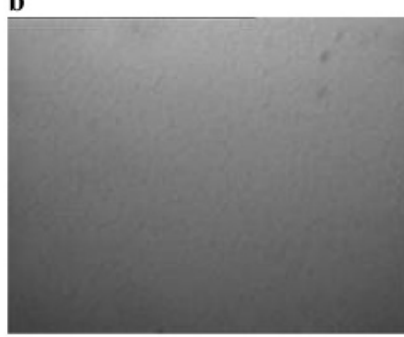

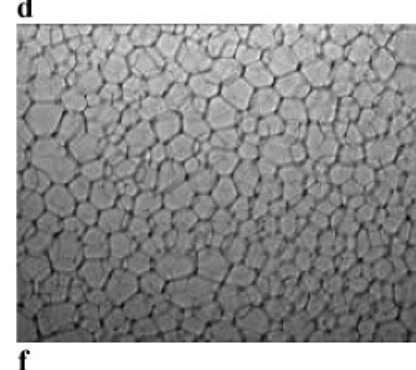

Figure 4. Sequence of optical micrographs $(a-f)$ obtained during the curing at $90{ }^{\circ} \mathrm{C}$ of DGEBA/5\% PMMA/PAMS sample, resulting in a final polygonal morphology. Full width is $125 \mu \mathrm{m}$. (g) SEM image of the surface showing packed pseudoregular polyhedral domains.

is also presented. Since only PMMA is labeled with the anthracene fluorescent probe (see Experimental Section for details), the image reveals that the thermoplastic forms a thin wall that surrounds the epoxy-rich particles being completely excluded from them. The TP is a continuous phase and the epoxy appears as nonconnected domains. Each phase itself is optically homogeneous since no variations in fluorescence intensity are observed. It is not possible to accurately determine the composition of the PMMA-rich phase, but the lack of fluorescence emission enables us to affirm that PMMA was completely absent from the polygonal domains.

The meaning of the observed morphology is that PMMA, which is perfectly soluble in DGEBA, finally becomes insoluble in the reacted mixture DGEBAPAMS. The mean size and distribution of the epoxy polygonal domains was relatively similar to the distribution of PAMS-rich domains present in the DGEBAPAMS mixtures at the beginning of polymerization, and this result evidences that curing progressed through DGEBA diffusion into the PAMS-rich droplets. From a detailed analysis of a representative surface area, the 


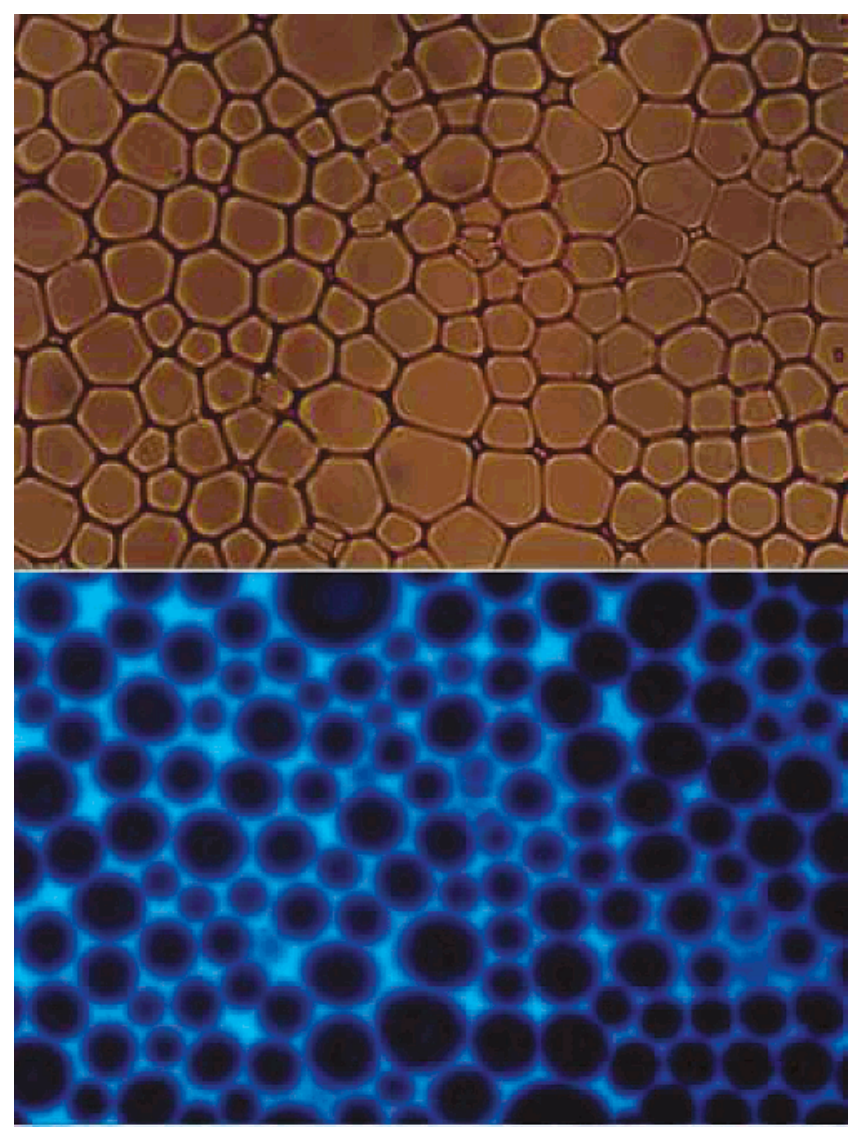

Figure 5. TOM (up) and epifluorescence (down) images of the fully cured system DGEBA/5\% PMMA/PAMS at $90{ }^{\circ} \mathrm{C}(\alpha$ $=0.92$ ). Full width is $125 \mu \mathrm{m}$. Fluorescence emission comes from the anthracene dye attached to the PMMA chain.

more frequent polygonal structure observed presented six faces (40\%), followed by five (32\%) and seven faces (13\%). The polygonal shape observed, which reflects the presence of three-dimensional polyhedral forms, as will be shown later, evidences the tendency of the system to undergo hexagonal close packing of distorted spheres as the volume fraction of the thermoset phase increased well above $\phi=0,74$, which belonged to close-packed spheres. ${ }^{31,32}$ The final structure of the material resembled the biliquid foams (aphrons) reported by Seb$\mathrm{ba}^{22}$ and suggests that surface forces govern the process.

At this point, we can ask ourselves why the PMMA films are stable and resist the compressive forces that would lead to coalescence between droplets through coarsening and breakup of the domains. Two facts must be considered. First, the PAMS droplets are cross-linked structures with restricted capacity to coalesce as reaction progresses, particularly in a system in which gelation appears at relatively low conversions. Second, the PMMA film can be considered to be a thin layer with two interfaces with the epoxy network. In both, the DGEBA chains (miscible with PMMA) will be orientated to the polymer film and will reduce interfacial tension, reducing film drainage to the low-pressure region focused on the triangular space where the polyhedron surfaces converge. ${ }^{22}$

Which component constitutes the matrix and which the dispersed phase is normally determined by the relative composition of the system with respect to the critical point. The critical composition before reaction can be roughly estimated using a thermodynamic model based on the Flory-Huggins approach, ${ }^{2}$ taking into

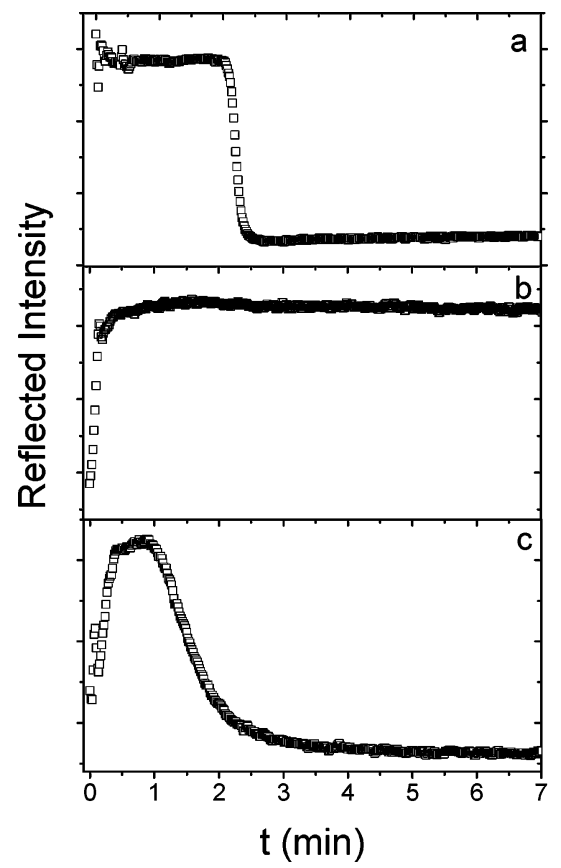

Figure 6. Intensity of the reflected light as a function of cured time at $90{ }^{\circ} \mathrm{C}$ for different reactive blends: (a) DGEBA/5\% PMMA/DAP; (b) DGEBA/PAMS; (c) DGEBA/5\% PMMA/ PAMS.

account the thermoplastic polydispersity but considering the epoxy-amine system as a pseudocomponent. It gives $\phi_{\text {crit,PMMA }}=6.7 \% \mathrm{v} / \mathrm{v}$ for DGEBA/PMMA/PAMS samples (6.0\% v/v for DGEBA/PMMA/DAP samples), as reported elsewhere in the literature for PMMA modified epoxides. ${ }^{20}$ Therefore, the model predicts, at least for $2 \%$ - and 5\%TP/DGEBA blends (corresponding to even lower $\varphi_{\text {TP }}$ on the ternary blend, see Table 3), PMMA segregation in a continuous thermoset matrix. The observation of the TP as continuous phase shows that the assumption of a pseudobinary epoxy/amine component can no longer be maintained in the system.

Analysis of the Transparency Window and Subsequent Morphological Evolution. Optical transparency is usually associated with the absence of domains with a characteristic length of the order of the light beam wavelength used in the experiment. The higher the wavelength used, the lower the sensitivity to incipient phase appearance or disappearance. It is preferable, from the practical point of view, to use visible light instead of infrared radiation, with the minimum possible wavelength to avoid absorption. Cloud points have been measured with visible radiation of $450 \mathrm{~nm}$, the minimum radiation level at which the sample does not absorb light. This is a common technique suitable for detecting domains of around $200 \mathrm{~nm}$ or even lower. Some examples of the reflected light intensity variation with reaction time at a curing temperature of $90^{\circ} \mathrm{C}$ are presented in Figure 6. For DGEBA/PMMA/DAP blends (Figure 6a), a single sharp transition corresponding to the phase separation of the thermoplastic was observed at roughly 2 min (before gel time, see Table 3). DGEBA/ PAMS binary blends displayed precisely the opposite trend. The fast reactive compatibilization between the two components increased the transparency of the sample. DGEBA/PMMA/PAMS blends showed more complex behavior. Several transitions were observed. Initially, the reflected light intensity increased to a maximum and then what appeared to be a transparency 


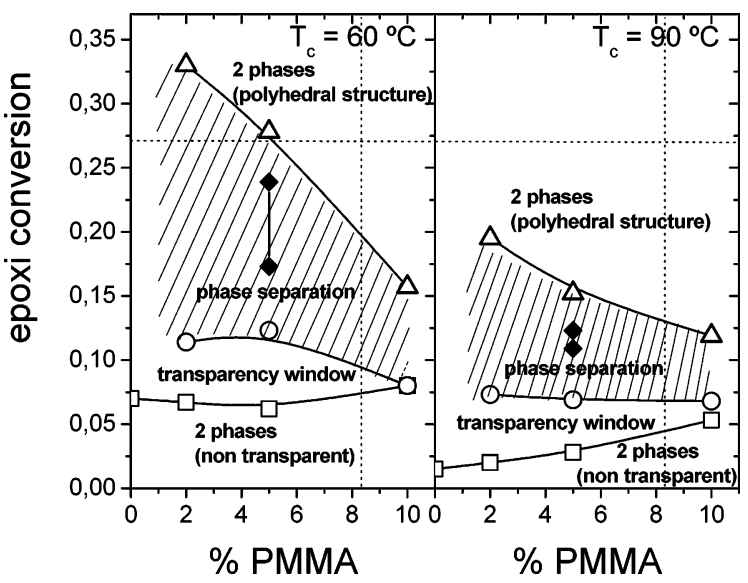

Figure 7. Epoxy conversion at the compatibilization ( $\square$ ), at the onset $(O)$ and offset $(\triangle)$ of cloud point curves measured by visible scattering obtained during the cure at 60 and $90{ }^{\circ} \mathrm{C}$ as a function of different \% PMMA on the epoxy precursor, for DGEBA/PMMA/PAMS samples. The horizontal and vertical dotted lines represent gel conversion and the theoretical critical PMMA composition respectively (see text for details). The shaded area shows the cloud point measurement interval. $(\diamond-\diamond)$ cloud point interval for DGEBA/PMMA/DAP.

window was maintained over a certain conversion range, until the biphasic structure observed in Figure $4 \mathrm{~g}$ was formed. As shown previously, these new phases comprised mainly PMMA and the thermosetting polymer. The decrease in light transmission, i.e., the cloud point, was gradual and took place in a longer time interval than that observed for the DGEBA/PMMA/DAP samples.

The time at which the reflected intensity reached a maximum was associated with the entrance into the transparency window. The time at which intensity began to decrease corresponded to the exit of the system from that window. The intersection between the two linear portions of the exit process can be taken as a measure of the end of the process. Each time corresponds to a conversion given by the time-conversion curve obtained by nIR. A diagram can be built plotting the conversion at which the different optical changes appear as a function of initial PMMA content on the epoxy precursor. These diagrams are shown in Figure 7 for two temperatures. The diagrams are completed with a horizontal line, showing the gel conversion. From examination of the diagrams, the following aspects become clear: (a) there is an optical solubility window whose location depends on the curing temperature, i.e., it appears at lower conversions at the higher curing temperature; (b) this window seems to close for high PMMA content, above $10 \%$ PMMA at $60{ }^{\circ} \mathrm{C}$; (c) the conversion at which the window appears increases with PMMA content, suggesting the delaying effect of PMMA on reactive compatibilization.

This diagram has been analyzed in terms of the optical properties of the curing system. It is known that maximum transparency is obtained for heterophasic systems, such as glass filled epoxy composites, when the refractive indices of both components match one another. ${ }^{33,34}$ It is therefore possible that, independently of the number of phases present (and the associated interfaces), the refractive indices of each phase become similar in the conversion range of interest (below 0.1), leading to optically transparent samples. Recalling Figure 5, it becomes clear that curing should proceed by incorporation of DGEBA units into the PAMS-rich droplets at least until the appearance of the transparency window.

The refractive index of each component of the reactive mixture was measured together with its dependency on temperature, and the corresponding data are presented in Table 2. As indicated in the Experimental Section, Table 2 also shows the calculated density and its dependency on temperature. The refractive indices of the species present in the mixture were similar enough, hence a linear additive law can be assumed for the refractive index, ${ }^{35,36}$

$$
n_{\text {phase }}=\Sigma n_{i} \phi_{i}
$$

where $\phi_{i}$ is the volume fraction of the $i$ component in the phase. This is a fairly good approach, as shown by the comparison of the refractive index measured for DGEBA/10\% PMMA, $n=1.554$, with the theoretically predicted index. Assuming that the reactive system consists of two phases, DGEBA/PMMA and DGEBA/ PAMS, it is possible to calculate when the refractive index of both phases will be equal, according to the following expression

$$
\begin{aligned}
& \left(n_{\mathrm{DGEBA}, T} \phi_{\mathrm{DGEBA}, 1}+n_{\mathrm{PMMA}, T} \phi_{\mathrm{PMMA}, 1}\right)_{\mathrm{phase} 1, \mathrm{~T}}= \\
& \left(n_{\mathrm{DGEBA}, T} \phi_{\mathrm{DGEBA}, 2}+n_{\mathrm{PAMS}, T} \phi_{\mathrm{PAMS}, 2}\right)_{\mathrm{phase} 2, T}
\end{aligned}
$$

where phase 1 and phase 2 correspond to the DGEBA/ PMMA and PAMS/DGEBA phases respectively, at a given temperature. To fulfill this condition, sufficient DGEBA molecules must diffuse through the interface into the PAMS-rich phase, increasing simultaneously the PMMA concentration on the TP-rich phase. A brief analysis shows that the two terms of the equation equals when the fraction of PAMS on the PAMS-rich phase reduces to approximately $26-28 \% \mathrm{v} / \mathrm{v}$ (small variations are due to the different curing temperatures and PMMA content). In terms of reactive functionalities, the primary amine hydrogen to epoxy equivalent ratio reaches the values $1.03-1.10$, i.e., fairly close to the stoichiometric ratio. On the other hand, DGEBA diffusion increase TP content on the PMMA/DGEBA phase by up to $35-39 \%$. These results show that the relative amount of DGEBA initially present in the TP-rich phase that have to diffuse to the PAMS-rich phase must be very high, between $86 \%$ for $10 \%$ PMMA blends and $97 \%$ for $2 \%$ PMMA blends.

The chemical reaction between DGEBA and PAMS initially takes place through the interdiffusion of DGEBA and PAMS chains across the interface between both components. This chemical process broadens the interface length and modifies the interfacial composition inducing a gradual change of the refraction index. The initial sharp interface becomes diffuse, scattering decreases, and the systems becomes clear and transparent. Nevertheless, the mechanism must be more complex since the high interaction parameter between PAMS and DGEBA, which can be deduced from the difference between the corresponding solubility parameters, precludes the mutual diffusion of both components. ${ }^{37}$ The process probably begins at the interface with a changing composition due to the progressive reaction between oxirane and amine groups. This composition facilitates diffusion of more DGEBA molecules through the interior of the PAMS-rich phase, increasing the amine functionalities accessible for reaction. Taking into account the functionality of PAMS, and according to Stockmayer 
condensation statistics, ${ }^{38,39}$ even at the very low conversions at which the solubility window appears $(\alpha=0.02-$ 0.08 , Figure 7), a considerable amount of the polyfunctional PAMS molecules have reacted with at least one DGEBA chain. As a result, the interfacial domain contains a large amount of low molecular weight PAMS chains grafted with DGEBA units that have two effects: they disrupt self-association of PAMS and increase its thermodynamic quality as a solvent of DGEBA. More DGEBA will be soluble in the PAMS-rich domains as a significant amount of DGEBA chains graft to the PAMS molecule. In other words, interfacial diffusion is so high that reaction kinetics is not diffusioncontrolled, as observed experimentally.

Fluorescence imaging (Figure 5) showed that PMMA was completely excluded from the thermoset phase; therefore, diffusion of PMMA into the PAMS-rich phase did not occur. This can be attributed to the increase in the mixing free energy as thermoset molecular weight increased. ${ }^{2}$ Before gelation, both the molecular weight and the molecular weight growing rate should be high for the polyaminosiloxane hardener used because of its polyfunctionality, which shortens the gel point. Therefore, possible PMMA diffusion through the interfacial domain, once enough DGEBA is on the PAMS-rich phase increasing their thermodynamic quality, will be delayed or even interrupted. Most PMMA is always excluded from the PAMS-rich phase, a completely homogeneous solution is never formed and there is not a critical conversion for phase separation. PMMA remains at the interfacial domain forming the observed thin skin, and this explains the absence of any influence on the kinetic results. As shown in Figure 7, the offset of the cloud point was higher for the system cured at the lower temperature. Again, this experimental finding cannot be explained in terms of simple thermodynamic arguments. In the hypothesis according to which the pseudobinary system presents the common UCST phase diagram (or even LCST), the predicted behavior should be the opposite. A more detailed examination of Figure 7 shows that the conversion interval in which phase separation appears was broader for the lower temperature; this suggests the issue of diffusion control. This interpretation is, in principle, consistent with two experimental findings presented above: the conversion at which apparent solubilization (actually optical transparency) appears increases with PMMA content and decreases with curing temperature. Both should be a consequence of increased viscosity and its variation with composition in the DGEBA/PMMA phase, in which TP content increases to $40 \%$, and this helps to explain the broad range in which the sample gradually became opaque. As viscosity increases, diffusion of DGEBA through the interface is hindered, although this effect decreases as curing temperature increases. It is therefore likely that viscosity may be so great that complete phase separation of PMMA (i.e., complete exclusion of DGEBA from the TP-rich phase) may be diffusioncontrolled. In other words, the DGEBA diffusion rate falls as TP concentration on the TP-rich phase increases; this effect was more appreciable for low curing temperatures or when the blend was highly concentrated in the TP component.

The above-mentioned description and interpretation of the morphology implies that the system must present two glass transition temperatures: one associated with the DGEBA/PAMS domains; and the other with the

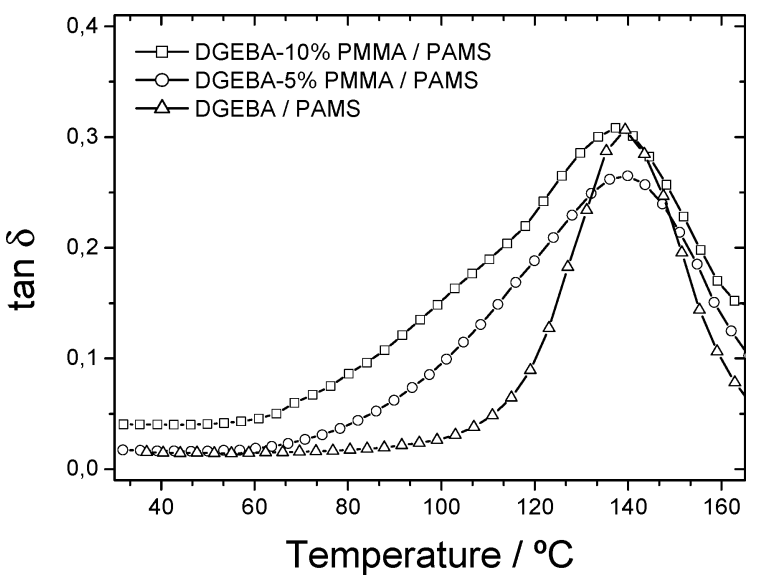

Figure 8. Loss factor (at $3 \mathrm{~Hz}$ ) as a function of temperature for the samples indicated in the graph. Curing at $90{ }^{\circ} \mathrm{C}, 1 \mathrm{~h}$ and postcured at $120^{\circ} \mathrm{C}$ for $2 \mathrm{~h}$.

PMMA-rich phase. Figure 8 shows the temperature variation of the loss factor for the neat system and for two compositions ( $5 \%$ and 10\%). To avoid overlapping of the thermal reactivation of the curing process (see Table 3), samples were postcured at $120^{\circ} \mathrm{C}$ for $2 \mathrm{~h}$. The three systems presented a similar relaxation in the high-temperature range, peaking at $140{ }^{\circ} \mathrm{C}$, which was associated with the glass transition temperature of the DGEBA/PAMS domains. As the amount of PMMA increased, a low temperature shoulder broadened the $\tan \delta$ peak. This shoulder was assigned to the relaxation of PMMA. Deconvolution of the peaks enabled us to estimate the glass transition temperature, which was $103{ }^{\circ} \mathrm{C}$, offering excellent agreement with the value measured by DSC presented in Table 1 . Therefore, we may now affirm that because no plasticization effects were observed, no significant amounts of DGEBA were present in the PMMA-rich phase; this agrees with the abovementioned calculus.

Morphology of Cured Blends. Influence of Temperature and PMMA Content. Figure 9 shows SEM images of DGEBA/PMMA/PAMS and DGEBA/PMMA/ DAP fracture surfaces, dry or chloroform etched to extract the PMMA. Fracture surfaces revealed the internal microstructure eliminating potential effects of the surfaces. In the micrograph shown in Figure 9a, the epoxy/PAMS domains now appear as spheroid granules covered by a thin skin and some filaments of the PMMA matrix. No cracks on the epoxy phase were observed; hence, the fracture extends mainly over the PMMA phase. In the central part of the image, a number of holes left by the pulled granules are clearly visible and confirm that PMMA glues the granules acting as the matrix. When the fracture surface was etched with a good PMMA solvent (Figure 9b) such as chloroform, the granules appeared free of any PMMA filament. In any case, it should be noted that after etching the granular structure did not collapse, indicating that granules must be interconnected to a certain extent. The structure was therefore cocontinuous. To confirm this interpretation, the surface of a number of etched granules was observed at a higher magnification (Figure 9c). Some fracture surfaces are easily visible on the granules and seem to correspond to flattened surface areas.

Another striking effect was observed when reducing PMMA content to 2\% (Figure 9d), changing the spheroid granules to larger faceted polyhedrons. This must be a consequence of the increase in the volume fraction of 

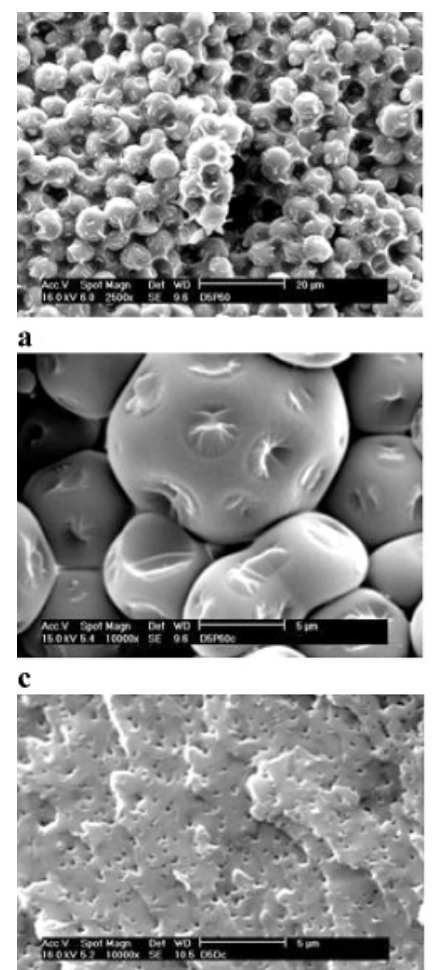

e

Figure 9. SEM micrographs of fracture surfaces: (a) DGEBA/ $5 \%$ PMMA/PAMS sample cured at $90{ }^{\circ} \mathrm{C}$; (b) the same sample as in part a but etched with chloroform for $24 \mathrm{~h}$ to remove PMMA; (c) detail of sample $b$ with more magnification; (d) DGEBA/2\% PMMA/PAMS sample cured at $120{ }^{\circ} \mathrm{C}$ and chloroform etched; (e) DGEBA/5\% PMMA/DAP sample cured at $90{ }^{\circ} \mathrm{C}$ and chloroform etched.

the thermoset phase, giving rise to flatter contact areas. The three-dimensional polyhedral structure is shown clearly here. This structure must also have been present in other samples, but an increase in TP content lead to more curved surfaces. The three-dimensional regular figure that fills a volume space best is the pentagonal dodecahedron. ${ }^{40}$ In the figure, many five-side faces are clearly present, particularly in larger particles. The presence of a number of six-side faces and four-side faces also suggest the presence of truncated octahedron polyhedrons, which can fill the space better (other irregular structures also present were due to the broad distribution in particle sizes). This polyhedral morphology is not usual for thermoset/thermoplastic blends at very low TP concentrations, because TP usually phase separates on dispersed droplets. It was obtained because of the impossibility to dissolve PMMA in the PAMSrich phase, whereas cross-linking with DGEBA progressed. Because of PAMS functionality, molecular weight increased at a faster rate than when conventional diamines were used. A micrograph showing the microstructure of DGEBA/5\% PMMA/DAP is presented for comparison purposes (Figure 9e). This system can be considered as the normal behavior of PMMA in a conventional epoxy/amine system. The etched fracture surface shows an evenly dispersed set of holes throughout the epoxy matrix. These holes, of about $0.25 \mu \mathrm{m}$ in diameter, were occupied by PMMA before etching. This quite different morphology arose because the surface tension of the matrix was high and because phase separation occurred far away from gelation.

The generated morphologies can be controlled by changing the blend composition and curing temperature.
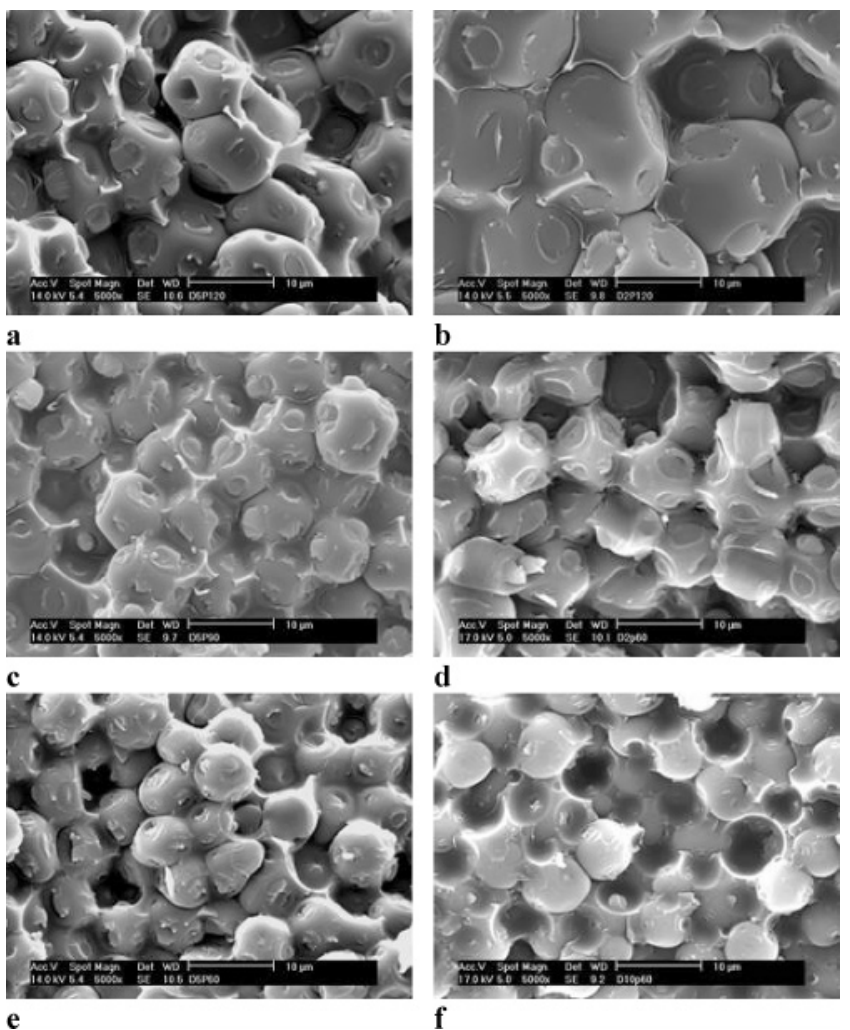

Figure 10. SEM micrographs of fracture surface: (a, c, and e) DGEBA/5\% PMMA/PAMS samples cured at 120, 90, and $60{ }^{\circ} \mathrm{C}$ respectively; (b and $\mathrm{d}$ ) DGEBA/2\% PMMA/PAMS samples cured at 120 and at $60{ }^{\circ} \mathrm{C}$ respectively; (f) DGEBA/ $10 \%$ PMMA/PAMS sample cured at $60{ }^{\circ} \mathrm{C}$.

Figure 10 shows some pictures of the morphologies achieved. When increasing the PMMA content in the initial blend with DGEBA, the mean size of the polyhedrons decreased. For example, at a curing temperature of $120^{\circ} \mathrm{C}$, mean size varied from $14.3 \mu \mathrm{m}$ (for $2 \%$ PMMA blends, Figure 10b) to $10.3 \mu \mathrm{m}$ (for 5\% PMMA blends, Figure 10a). This behavior was observed at the three curing temperatures analyzed, although size decreased to a lesser extent when curing temperature was low: from $7.9 \mu \mathrm{m}$ for $2 \%$ PMMA blends to $6.3 \mu \mathrm{m}$ for 5\% PMMA blends, and to $5.6 \mu \mathrm{m}$ for 10\% PMMA blends (Figure 10, parts d, e, and f). It is interesting to observe that the shape of the thermoset polyhedron became more spherical as PMMA content increased, and the interconnected surfaces seemed to fall. It is likely that at a certain PMMA content level, above $10 \%$ on the epoxy precursor, the thermoset phase will be formed by nonconnected, dispersed droplets, i.e., in a phaseinverted morphology.

When PMMA content was fixed, the mean size of the thermoset connected globules increased by raising the curing temperature (see pictures on the left-hand side in Figure 10). Therefore, for 5\% PMMA samples, the mean size varied from $6.2 \mu \mathrm{m}$ (for $60{ }^{\circ} \mathrm{C}$ curing, Figure $10 \mathrm{e}$ ) to $8.2 \mu \mathrm{m}$ (for $90^{\circ} \mathrm{C}$ curing, Figure 10c) and to 10.3 $\mu \mathrm{m}$ (for $120{ }^{\circ} \mathrm{C}$ curing, Figure 10a). It is clear that morphology can be controlled between certain limits, at least between 15 and $5 \mu \mathrm{m}$. The decrease in the mean particle size of the polyhedral epoxy phases when PMMA content increased and/or curing temperature decreased was probably due to a viscosity effect. Increasing PMMA content prompts an excessive increase in the viscosity of the initial epoxy-rich phase and decreasing the curing temperature increases the viscos- 
ity of each phase. Higher viscosity levels reduced the coalescence of PAMS droplets, which acted as the nucleus of the morphology observed. This morphology can be also observed with other thermoplastic polymers (polysulfone, polystyrene) if an immiscible hardener (both with epoxy precursor and TP), i.e., PAMS, is used. This will be studied in future research.

\section{Conclusions}

The behavior of PMMA/DGEBA blends with a polyamine functional hardener (PAMS) was studied in different curing conditions and with different PMMA contents, up to $10 \% \mathrm{w} / \mathrm{w}$ on the DGEBA precursor. At the TP percentages analyzed in this study, TP did not influence the kinetics of the curing process, but it had a strong effect on the completion of reactive compatibilization. At a certain conversion level, an optically transparent window was reached, mainly due to optical effects. In other words, the absence of clear interfaces rather than homogeneity is responsible for the transparency window appearance. The transparency window was delayed as PMMA content increased, showing that reactive compatibilization was hindered because the process was diffusion-controlled in the last stage, when the PMMA concentration in the TP-rich phase was high. The PMMA solution in the cross-linked DGEBA/PAMS thermoset phase was thermodynamically unfavorable during all the process.

A cocontinuous morphology is observed on the cured materials, with the thermoplastic forming a continuous skin surrounding an epoxy-rich interconnected polyhedral structure, even for very low TP content. The development of this morphology should not be explained by thermodynamical criteria based on pseudobinary epoxy/amine system and the analysis of the mixing energy with PMMA, because thermoplastic was not soluble in the reactive polyaminosiloxane polymer from the beginning, and the initial mixture was heterogeneous. Instead, the morphology originated in the earlier stages of the compatibilization process, when the amine network precursor coalesced in a DGEBA/PMMA continuous matrix. As DGEBA diffused into the PAMS-rich phase, PMMA formed a thin surrounding film. According to the results obtained in this research study and previous work, the control of initial reactive compatibilization of immiscible network precursors is a key for achieving controlled cocontinuous morphologies in thermoset/TP blends, independently of thermoplastic content.

Acknowledgment. The authors would like to express their gratitude to the Epoxsil (MAT2000-0391P4-02) and Fibrodont (MAT2001-0677-P3) projects for financial support. The authors would also like to thank to Dr. I. Esteban (UNED) for his assistance with the TOM micrographs and to Dr. J. Iruin (UPV) for his assistance with the density measurements.

\section{References and Notes}

(1) May, C. A. Epoxy resins. Chemistry and Technology; Marcel Dekker: New York, 1988

(2) Williams, R. J. J.; Rozenberg, B. A.; Pascault, J. P. Adv. Polym Sci. 1997, 128, 95.
(3) (a) Kinloch, A, J. In Rubber-Toughened Plastics, Riew, C. K., Ed., ACS, Washington, 1989. (b) Toughened Plastics I: Science and Engineering; Kinloch, A. J., Riew, C. K., Eds., American Chemical Society: Washington, DC, 1993.

(4) Pascault, J. P.; Sautereau, H.; Verdu, J.; Williams, R. J. J. Thermosetting Polymers; Marcel Dekker: New York, 2002; Chapter 13 and references therein.

(5) Pascault, J. P.; Williams, R. J. J. In Polymer Blends, Paul, D. R., Bucknall, C. B., Eds.; Wiley: New York, 1999; Vol. 1 Chapter 13.

(6) Loera, A. G.; Cara, F.; Dumon, M.; Pascault, J. P. Macro-molecules $2002,35,6291$.

(7) Gubbels, F.; Blacher, S.; Vanlathem, E.; Jerome, R.; Deltour, R.; Browers, F.; Teyssie, P. Macromolecules 1995, 28, 1559.

(8) Venderbosch, R. W.; Meijer, H. E. H.; Lemstra, P. Polymer 1994, 35, 4349.

(9) Kinloch, A. J.; Yuen, M. L.; Jenkins, S. D. J. Mater. Sci. 1994, 29, 3781.

(10) Hedrick, J. L.; Yilgor, I.; Wilkes, G. L.; McGrath, J. E. Polym. Bull. (Berlin) 1985, 13, 201.

(11) Prolongo, S. G.; Cabanelas, J. C.; Fine, T.; Pascault, J. P. J. Appl. Polym. Sci. 2004, in press.

(12) Norton, L. J.; Smigolova, V.; Pralle, M. U.; Hubenko, A.; Dai, K. H.; Kramer, E. J.; Hahn, S.; Berglund, C.; Desoven, B. Macromolecules 1995, 28, 1999.

(13) Cabanelas, J. C.; Serrano, B.; Gonzalez-Benito, J.; Bravo, J.; Baselga, J. Macromol. Rapid Commun. 2001, 22, 694.

(14) Prolongo, S. G.; Cabanelas J. C.; Baselga, J.Macromol. Symp. 2003, 198, 203.

(15) Cabanelas, J. C.; Prolongo, S. G.; Serrano, B.; Bravo, J.; Baselga, J. J. Mater. Proc. Technol. 2003, 143-144, 311.

(16) Ochi, M.; Shimaoka, S. Polymer 1999, 40, 1305.

(17) Gámez, C. M.; Bucknall, C. B. Polymer 1993, 34, 2111.

(18) Woo, E. M. Wu, M. N. Polymer 1996, 37, 2485.

(19) Ritszenthaler, S.; Girard-Reydet, E.; Pascault, J. P. Polymer 2000, 41, 6375.

(20) Remiro, P. M.; Marieta, C.; Riccardi, C. C.; Mondragón, I. Polymer 2001, 42, 9909.

(21) Janarthanan, V.; Thyagarajan, G. Polymer 1992, 33, 3593.

(22) Sebba, F. Foams and Biliquid Foams-Aphrons; Wiley: New York, 1987.

(23) Serrano, B.; Baselga, J.; Bravo, J.;. Mikes, F.; Sese, L.; Esteban, I.; Pierola, I. F. J. Fluoresc. 2000, l10, 135.

(24) Kortaberria, G.; Arruti, P.; Gabilondo, N.; Mondragón, I. Eur. Polym. J. 2004, 40, 129.

(25) Serrano, B.; Levenfeld, B.; Bravo, J.; Baselga, J. Polym. Eng. Sci. 1996, 36,175 .

(26) Varley, R. J.; Hodgkin, J. H.; Hawthorne, D. G.; Simon, G. P.; McCulloch, D. Polymer 2000, 41, 3425.

(27) Bonnet, A.; Pascault, J. P.; Sauterau, H.; Taha, M.; Camber-lin, Y. Macromolecules 1999, 32, 8517.

(28) Grulke, E. A. In Polymer Handbook, 4th ed.; Brandrup, J., Immergut, E. J., Grulke, E. A., Eds.; Wiley: New York, 1999; Chapter VII.

(29) Pozuelo, J.; Baselga, J. Polymer 2002, 43, 6049.

(30) Bhargawa, R.; Wang, S.-Q.; Koenig, J. L. Macromolecules 1999, 32, 8989.

(31) Princen, H. M. J. Colloid. Interface Sci. 1979, 71, 55.

(32) Adamson, A. W. Physical Chemistry of Surfaces, 5th ed.; Wiley: New York, 1990.

(33) Sato, H.; Iba, H.; Naganuma, T.; Kagawa, Y. Philos. Mag. 2002 B82, 1369 .

(34) Naganuma, T.; Kagawa, Y. J. Mater. Sci. 2003, 38, 3103.

(35) Wedgewood, A. R.; Seferis, J. C. Polym. Eng, Sci. 1984, 24, 328.

(36) Seferis, J. C.; Samuels, R. J. Polym. Eng. Sci. 1979, 19, 975.

(37) Ajji, A. In Polymer Blends Handbook; Utracki, L. A., Ed.; Kluwer: The Netherlands, 2002; Vol. 1.

(38) Stockmayer, W. H. J. Polym. Sci. 1952, 9, 69.

(39) Stockmayer, W. H. J. Polym. Sci. 1953, 11, 424.

(40) Matzke, E. B. Cited in ref 32.

(41) Wunderlich, W. In Polymer Handbook, 4th ed., Brandrup, J.; Inmergut, E. H.; Grulke, A. Eds.; Wiley: New York, 1999; p V-87. 